\title{
Checklist de Bombyliidae (Diptera) no Estado do Mato Grosso do Sul
}

\author{
Carlos José Einicker Lamas \& Carolina Yamaguchi ${ }^{1,2}$
}

\begin{abstract}
1. Museu de Zoologia da Universidade de São Paulo, Av. Nazaré, 481, Ipiranga, 04263-000, São Paulo, SP, Brasil. (einicker@usp.br) 2. Instituto de Biociências USP, Rua do Matão, Trav. 14, n 321, Cidade Universitária, 05508-090, São Paulo, SP, Brasil.
\end{abstract}

Recebido 28 novembro 2016

Aceito 6 fevereiro 2017

DOI: $10.1590 / 1678-4766 e 2017133$

\begin{abstract}
Checklist of Bombyliidae (Diptera) from Mato Grosso do Sul state. Bombyliidae is one of the largest Diptera families with more than 4,500 worldwide species. Their species vary from robust to thin, and may be small to large (2-20 mm) and looks like bees or wasps. Adults can often be seen either resting and sunning themselves on trails, rocks or twigs or feeding on flowering plants as they are nectar feeders. All known larvae of bee flies are predators or parasitoids of arthropods. The Brazilian fauna of bombyliids comprises at the moment 110 species of which 12 have been recorded to the state of Mato Grosso do Sul.
\end{abstract}

KEYWORDS. Taxonomy, diversity, Homoeophthalmae, Tomophthalmae, Biota-MS Program.

RESUMO. Bombyliidae é uma das maiores famílias de Diptera com mais de 4.500 espécies conhecidas em todo o mundo. Suas espécies variam de robustas a delgadas e podem ser de diminutas a muito grandes (2-20 mm) e parecem abelhas ou vespas. Adultos são comumente avistados pousados ou tomando sol em trilhas, pedras ou galhos, ou ainda se alimentando de néctar em flores. Todas as larvas conhecidas de Bombyliidae são predadoras ou parasitoides de artrópodes. A fauna brasileira de Bombyliidae compreende, até o momento, 110 espécies das quais 12 possuem registro assinalado para o estado do Mato Grosso do Sul.

PALAVRAS-CHAVE. Taxonomia, diversidade, Homoeophthalmae, Tomophthalmae, Programa Biota-MS.

Os bombiliídeos pertencem a umas das maiores famílias de Diptera, incluindo mais de 4.500 espécies (Evenhuis \& GREATHEAD, 1999). São amplamente distribuídos pelo mundo, ocorrendo em todos os continentes, exceto no Antártico (Hull, 1973), embora sendo mais abundantes nas regiões temperadas (MCALPINE, 1981).

Os adultos da maioria das espécies apresentam asas grandes em proporção ao corpo (HuLL, 1973), tornando-os voadores ágeis e poderosos, concorrendo com as moscas da família Syrphidae nas habilidades de pairar e mover em todas as direções enquanto voam (EvEnHUIS \& GREATHEAD, 1999). Os adultos se alimentam de néctar (exceto em alguns gêneros com aparelho bucal não funcional como a Oestranthrax), e as fêmeas, além do néctar, utilizam também o pólen de angiospermas que é um nutriente indispensável para o desenvolvimento dos ovos. Como resultado deste tipo de alimentação, os Bombyliidae são considerados os principais polinizadores de muitas plantas, especialmente daquelas com ocorrência nas regiões mais áridas do planeta (EvENHUIS \& GreatheAD, 1999).

\section{MATERIAL E MÉTODOS}

A lista de espécies de Bombyliidae registradas para o Mato Grosso do Sul foi elaborada com base no catálogo de Evenhuis \& Greathead (1999). Bibliografia adicional consultada: D'Andretta \& CARrera (1950), PAinter et al. (1978), Lamas \& Couri (1999), Evenhuis \& Greathead (2003), CunHa et al. $(2007,2009)$ e LAMAS \& YAMAGUCHI (2010).

\section{RESULTADOS}

Lista de espécies de Bombyliidae registradas no Estado do Mato Grosso do Sul.

\section{DIVISÃO HOMOEOPHTHALMAE}

BOMBYLIINAE

Heterostylum ferrugineum Fabricius, 1805. Localidade-tipo: “Americae Insulis”. Distribuição: Bolívia, Brasil [Bahia, Ceará, Mato Grosso do Sul (Porto Murtinho), Roraima, São Paulo], British Virgin Island, Paraguai, Puerto Rico, U. S. Virgin Island.

Euprepina maracajula Hull, 1971. Localidade-tipo: Brasil, Mato Grosso (= Mato Grosso do Sul). Distribuição: Brasil [Mato Grosso do Sul (Maracajú)].

Euprepina nuda Hull, 1971. Localidade-tipo: Brasil, Santa Catarina. Distribuição: Argentina, Brasil [Mato Grosso do Sul (Maracajú), Minas Gerais, Rio de Janeiro, Rio Grande do Sul, Santa Catarina, São Paulo]. Neodischistus collaris Painter, 1933. Localidade-tipo: Argentina. Distribuição: Argentina, Bolívia, Brasil [Mato Grosso, Mato Grosso do Sul (Corumbá, Porto Murtinho), Paraná, São Paulo] e Paraguai. Neodischistus marstoni Lamas \& Yamaguchi, 2010. Localidade-tipo: Brasil, São Paulo, Araçatuba. Distribuição: Argentina, Brasil [Ceará, Goiás, Espirito Santo, Maranhão, Mato Grosso, Mato Grosso do Sul 
(Maracajú, Três Lagoas, Porto Murtinho), São Paulo].

TOXOPHORINAE

Systropus nitidus Wiedemann, 1830. Localidade-tipo: "Brasilia" (= Brasil ou Uruguai). Distribuição: Brasil (Amapá, Goiás, Mato Grosso, Mato Grosso do Sul, Minas Gerais, Pará, Paraná, Rio de Janeiro, Santa Catarina, São Paulo).

Toxophora aurea Macquart, 1848. Localidade-tipo: Brasil, Paraná. Distribuição: Brasil [Acre, Amapá, Amazonas, Distrito Federal, Mato Grosso, Mato Grosso do Sul (Porto Murtinho), Pará, Paraná, Rio de Janeiro, Roraima, São Paulo], Guiana Francesa, Paraguai, Suriname. Toxophora cuprea Fabricius, 1787. Localidade-tipo: "Cajennae" (=Guiana Francesa). Distribuição: Brasil [Goiás, Mato Grosso do Sul (Salobra), Pará], Colômbia, Guiana Francesa, México, Panamá, Suriname.

Toxophora leucon Séguy, 1930. Localidade-tipo: Argentina. Distribuição: Argentina; Bolívia; Brasil [Mato Grosso do Sul (Corumbá), Pará, São Paulo]; Paraguai.

\section{DIVISÃO TOMOPHTHALMAE}

ANTHRACINAE

Anthrax cephus Fabricius, 1805. Localidade-tipo: "America Meridionali". Distribuição: Brasil (Amazonas, Goiás, Mato Grosso, Mato Grosso do Sul, Minas Gerais, Paraná, Rio de Janeiro, Santa Catarina, São Paulo), Costa Rica, Panamá.

Anthrax trimaculatus Macquart, 1848. Localidade-tipo: "Brasil" (= Brasil ou Uruguai). Distribuição: Argentina, Brasil (Bahia, Ceará, Mato Grosso do Sul, Minas Gerais, Paraíba, Pernambuco, Rio Grande do Sul, São Paulo), Panamá, Uruguai, Venezuela.

Hyperalonia morio morio (Fabricius, 1775). Localidade-tipo: "Brasilia" (= Brasil ou Uruguai). Distribuição: Argentina, Bolívia, Brasil (Amazonas, Ceará, Espírito Santo, Goiás, Mato Grosso, Mato Grosso do Sul, Minas Gerais, Paraná, Rio de Janeiro, Rio Grande do Sul, Rondônia, Santa Catarina, São Paulo), Chile, Paraguai e Uruguai.

Comentários sobre a lista e riqueza do estado comparado com outras regiões. Levando em conta que existem 110 espécies de moscas da família Bombyliidae com ocorrência assinalada para o Brasil, o número de registros encontrados para o estado do Mato Grosso do Sul equivale a cerca de $10 \%$ destes registros. Embora não haja outro levantamento semelhante para os demais estados brasileiros, é possível presumir que os valores aqui observados colocam o Mato Grosso do Sul entre os estados com o melhor conhecimento da fauna desta família. Estes números ainda são, com absoluta certeza, bastante subestimados, entretanto mostram que houve, no passado, um esforço por parte de alguns entomólogos em formar coleções. Para esta família, destacam-se, principalmente, eventos de coleta realizados na localidade de Salobra, próximo à cidade de Miranda, Maracajú e Porto Murtinho.

Principais grupos de pesquisa. $\mathrm{O}$ principal grupo de pesquisa em Sistemática e Taxonomia de Bombyliidae no Brasil encontra-se no Museu de Zoologia da Universidade de São Paulo, sob a coordenação do Dr. Carlos Lamas. A seguir, apresentamos uma lista dos taxonomistas capacitados a identificar exemplares do Brasil. Essa lista foi construída com base nos trabalhos publicados sobre taxonomia de Bombyliidae envolvendo táxons da fauna neotropical: André Mallemont Cunha, Museu Nacional UFRJ, Rio de Janeiro, RJ, Brasil; Carolina Yamaguchi, Museu de Zoologia da Universidade de São Paulo, São Paulo, SP, Brasil; Paula Fernanda Motta Rodrigues, Museu de Zoologia da Universidade de São Paulo, São Paulo, SP, Brasil; Neal
Luit Evenhuis, J. Linsley Gressitt Center for Research in Entomology, Bishop Museum, Honolulu, Hawaii, EUA.

Principais acervos. Com base nos dados levantados por CARVALHO et al. (2002), as quatro principais instituições detentoras de acervo da família Bombyliidae no Brasil são: Museu Nacional do Rio de Janeiro (MNRJ); Museu de Zoologia da Universidade de São Paulo (MZUSP); Departamento de Zoologia da Universidade Federal do Paraná (DZUP) e Instituto Nacional de Pesquisas da Amazônia (INPA).

Principais lacunas de conhecimento. Podemos afirmar que, comparativamente, o único bioma brasileiro razoavelmente melhor conhecido, para a área de diversidade e sistemática de Diptera, é a Mata Atlântica no eixo RioSão Paulo. Por outro lado, o conhecimento disponível até o momento para o restante da Mata Atlântica e para todos os demais biomas está bastante aquém do desejado e do necessário.

Perspectivas de pesquisa para os próximos 10 anos. Para as pesquisas envolvendo Diptera, particularmente Bombyliidae, existe uma grande expectativa relacionada ao desenvolvimento do projeto "Rede temática para estudos de diversidade, sistemática e limites distribucionais de Diptera nos estados do Mato Grosso, Mato Grosso do Sul e Rondônia", aprovado no âmbito do Edital SIBIOTA, em uma parceria do CNPq com a FAPESP. O projeto teve início em fevereiro de 2010 e vem amostrando, de forma continuada, localidades estratégicas nestes três estados. Sendo assim, espera-se que haja um aumento bastante significativo no conhecimento da diversidade de dípteros, incluindo Bombyliidae, nos próximos anos.

Agradecimentos. À Fundação de Apoio ao Desenvolvimento do Ensino, Ciências e Tecnologia do Estado de Mato Grosso do Sul (Fundect) e à Superintendência de Ciências e Tecnologia do Estado de Mato Grosso do Sul (Sucitec/MS) pelo convite de participação neste fascículo especial da Iheringia, Série Zoologia e o suporte financeiro para sua publicação. Ao Conselho Nacional de Desenvolvimento Científico e Tecnológico (CNPq) (Proc. 563256/2010-9) e Fundação de Amparo à Pesquisa do Estado de São Paulo (FAPESP) (Proc. 2010/52314-0) pelo auxílio conferido ao projeto "Rede temática para estudos de diversidade, sistemática e limites distribucionais de Diptera nos estados do Mato Grosso, Mato Grosso do Sul e Rondônia", aprovado no âmbito do Edital SIBIOTA.

\section{REFERÊNCIAS BIBLIOGRÁFICAS}

D’Andretta, M. A. V. \& Carrera, M. 1950. Sobre as espécies brasileiras de Toxophorinae (Diptera, Bombyliidae). Dusenia 1(6):351-374.

Carvalho, C. J. B.; Couri, M. S.; Toma, R.; Rafael, J. A.; Harada, A. Y.; Bonatto, S. R.; Henriques, A. L. \& Gastal, H. A. De O. 2002. Principais coleções brasileiras de Diptera: Histórico e situação atual. Monografías Tercer Milenio 2:37-52.

Cunha, A. M.; Lamas, C. J. E. \& Couri, M. S. 2007. Revision of the New World bee fly genus Heterostylum Macquart (Diptera, Bombyliidae, Bombyliinae). Revista Brasileira de Entomologia 51(1):12-22.

Cunha, A. M.; Lamas, C. J. E. \& Couri, M. S. 2009. Redescription of two Neotropical species of Toxophora Meigen (Diptera, Bombyliidae, Toxophorinae). Papéis Avulsos de Zoologia 49(18):221-227.

Evenhuis, N. L. \& Greathead, D. J. 1999. World catalog of bee flies (Diptera Bombyliidae). Leiden, Backhuys Publishers. 75p.

Evenhuis, N. L. \& Greathead, D. J. 2003. World catalog of bee flies (Diptera: Bombyliidae): Corrigenda and addenda. Zootaxa 300:1-64. 
Hull, F. M. 1973. The beeflies of the world. The genera of the family Bombyliidae. Bulletin of the United States National Museum 286:3687.

Lamas, C. J. E. \& Couri, M. S. 1999. Revisão de Euprepina Hull, 1971 (Diptera, Bombyliidae, Bombyliinae). Revista Brasileira de Zoologia 16(2):461-482.

Lamas, C. J. E. \& Yamaguchi, C. 2010. Revision of Neodischistus Painter, 1933 (Diptera, Bombyliidae, Bombyliinae). Zootaxa 2540:48-58.
McAlpine, J. F. 1981. Morphology and terminology - Adults. In: McAlPine, J. F.; Peterson, B. V.; Shewell, G. E.; Teskey, H. J.; Vockeroth, J. R. \& Wood, D. M. eds. Manual of Neartic Diptera. Ottawa, Research Branch, Agriculture Canada. Monograph, 27. 674p.

Painter, R. H.; Painter, E. M. \& Hall, J. C. 1978. Family Bombyliidae. A Catalogue of the Diptera of the Americas South of the United States 38:1-92. 